\title{
Registros de enfermagem como ferramenta para a gerência do cuidado clínico-hospitalar
}

\author{
Nursing records as a tool for hospital clinical care management \\ Los registros de enfermería como herramienta para la gestión de la atención clínica hospitalaria
}

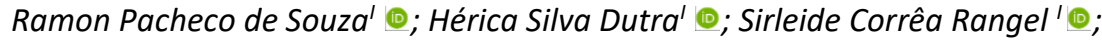

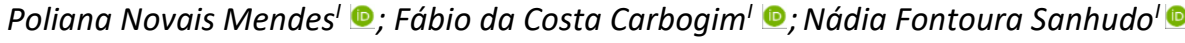

\begin{abstract}
'Universidade Federal de Juiz de Fora, Juiz de Fora, MG, Brasil
RESUMO

Objetivo: compreender a utilização dos registros de enfermagem na gerência do cuidado clínico-hospitalar. Método: estudo qualitativo, de caráter compreensivo, realizado em um hospital público de ensino de Minas Gerais, com 13 enfermeiras. Os dados foram coletados por entrevistas semiestruturadas, observação participante e pesquisa documental, entre outubro e novembro de 2017, e tratados pelo software IRaMuTeQ e análise de conteúdo. Protocolo de pesquisa aprovado pelo Comitê de Ética em Pesquisa da instituição. Resultados: após análise, emergiram três categorias: contexto de utilização dos registros e o desenvolvimento da gerência do cuidado; instrumentos adotados na elaboração dos registros; registros de enfermagem como meio de comunicação. Conclusão: os registros de enfermagem contribuem para o gerenciar e o cuidar, de maneira articulada. Entretanto, por meio da observação participante, constatou-se que os registros de enfermagem estavam associados apenas ao gerenciar, desvinculados do cuidar, o que dificulta a utilização destes como ferramenta de comunicação interprofissional e evidência da qualidade do cuidado.
\end{abstract}

Descritores: Enfermagem; Serviço Hospitalar de Enfermagem; Registros de Enfermagem; Organização e Administração.

\begin{abstract}
Objective: to understand the use of nursing records in hospital clinical care management. Method: this comprehensive qualitative study was conducted with 13 nurses at a public teaching hospital in Minas Gerais State, Brazil. Data were collected by semi-structured interviews, participant observation, and documentary research, between October and November 2017. The data were processed by IRaMuTeQ software and content analysis. The study was approved by the research ethics committee. Results: three categories emerged: the context in which the records are used and how care management takes place; the instruments adopted in preparing the records; and nursing records as a means of communication. Conclusion: nursing records contribute to management and to care, interrelatedly. However, participant observation made it evident that the nursing records were associated only with managing, and were divorced from care, making it difficult to use them as a tool for interprofessional communication and as evidence of the quality of care.
\end{abstract}

Descriptors: Nursing; Nursing Service, Hospital; Nursing Records; Organization and Administration.

\section{RESUMEN}

Objetivo: comprender el uso de los registros de enfermería en la gestión de la atención clínica hospitalaria. Método: estudio cualitativo y exhaustivo, realizado en un hospital público de enseñanza en Minas Gerais, junto a 13 enfermeras. Se recolectaron los datos mediante entrevistas semiestructuradas, observación participante e investigación documental, entre octubre y noviembre de 2017. Los datos fueron procesados por el software IRaMuTeQ y el análisis de contenido. El estudio fue aprobado por el Comité de Ética de la Investigación. Resultados: después del análisis, surgieron tres categorías: contexto de utilización de los registros y desarrollo de la gestión del cuidado; instrumentos adoptados en la elaboración de los registros; registros de enfermería como medio de comunicación. Conclusión: los registros de enfermería contribuyen a la gestión y el cuidado, de forma articulada. Sin embargo, a través de la observación participante, se hizo evidente que los registros de enfermería estaban asociados apenas con la gestión, sin relación con el cuidado, lo que dificulta su utilización como herramienta de comunicación interprofesional y evidencia de la calidad del cuidado.

Descriptores: Enfermería; Servicio de Enfermería en Hospital; Registros de Enfermería; Organización y Administración.

\section{INTRODUÇÃO}

Os registros efetuados no prontuário do paciente constituem um documento legal importante na defesa dos profissionais em caso de processos jurídicos e éticos. Além da evidência legal, possuem outras finalidades: compartilhamento de informações entre a equipe de enfermagem e demais profissionais, planejamento do cuidado, avaliação da qualidade da assistência, fonte de dados para ensino e pesquisa, auditoria e relatório permanente dos cuidados oferecidos ${ }^{1}$.

Agradecemos à Universidade Federal de Juiz de Fora, Brasil, pelo apoio financeiro a esta pesquisa.

Autora correspondente: Sirleide Corrêa Rangel. E-mail: sirleiderangel@gmail.com

Editora Científica: Cristiane Helena Gallasch; Editor Associado: Sergio Correa Marques 
Entretanto, a fragilidade nos registros de enfermagem ocorre em muitas instituições e está relacionada, principalmente, a: não utilização do carimbo, ausência de assinatura do profissional, rasuras, escrita ilegível e espaços em branco ${ }^{2}$. Além disso, a utilização de abreviaturas não padronizadas pode suscitar interpretações equivocadas, expondo o paciente a riscos, e comprometer a continuidade do serviço ${ }^{3}$. O uso de siglas, abreviaturas não convencionais e nomes comerciais de artefatos como Abbocath ${ }^{\circledR}$ se referindo a cateter venoso também foi observado em outro estudo 4 .

Registros superficiais, incompletos, não retratando a realidade do paciente e as intervenções de enfermagem realizadas dificultam a identificação do Processo de Enfermagem (PE), contribuindo para a invisibilidade profissional ${ }^{5}$. Auditorias em prontuários evidenciaram que os registros ainda são incipientes e que há uma relação positiva entre registros de enfermagem e qualidade do cuidado prestado ${ }^{6}$. Registros acurados permitem que os dados se transformem em informações pertinentes para o planejamento do cuidado. Sendo assim, mediações e intervenções educativas aplicadas a fim de sensibilizar e instruir os profissionais quanto à importância da clareza dos registros podem ajudar a aperfeiçoar a qualidade das anotações e impactar a continuidade de um cuidado seguro ${ }^{3,7,8}$.

Desse modo, os enfermeiros têm a responsabilidade de garantir que os registros sejam precisos e completos, como ferramenta para o gerenciamento do cuidado ${ }^{9}$. Entende-se o registro de enfermagem como parte inerente da gerência do cuidado, à medida que envolve aspectos relacionados às dimensões gerenciar/administrar e cuidar/assistir, como unidades inseparáveis ${ }^{10}$.

Exercer a gerência do cuidado de enfermagem torna-se uma ação complexa baseada em multidimensões que podem envolver os aspectos individual, familiar, organizacional, sistêmico e societário, que abrange a sociedade civil e o estado, portanto o enfermeiro precisa estabelecer relações interativas entre todas essas dimensões para gerenciar o cuidado ${ }^{11}$.

A Agenda Nacional de Prioridades de Pesquisa em Saúde do Ministério da Saúde aponta como prioridade de pesquisa em enfermagem na Região das Américas, no tópico ligado a Estrutura, Organização e Dinâmica dos Sistemas e Serviços de Saúde, a gestão e intervenções de enfermagem no cuidado individual e coletivo em saúde ${ }^{12}$.

Discutir sobre os registros de enfermagem é algo recorrente, entretanto a sua utilização como uma ferramenta para a gerência do cuidado carece de mais evidências científicas. Estudos com essa abordagem são necessários para os enfermeiros repensarem e ressignificarem sua prática de maneira a concretizarem o registro de enfermagem como sustentação para a gerência do cuidado. Logo, fundamentado no pensamento complexo de Edgar Morin ${ }^{13}$, este estudo tem como objetivo compreender a utilização dos registros de enfermagem na gerência do cuidado hospitalar.

\section{REFERENCIAL TEÓRICO}

O Pensamento Complexo formulado por Edgar Morin ${ }^{13}$ foi considerado como alicerce para este estudo, para uma melhor compreensão da gerência do cuidado de enfermagem e suas relações e interações com os registros de enfermagem. Nessa visão teórico-filosófica, o cuidado de enfermagem não pode ser configurado como atitude simplificadora e reducionista, mas como uma construção que precisa de reflexões, interações e autoconhecimento ${ }^{14}$. Sob esse enfoque, o cuidado deve considerar o ser humano em sua unidade e pluralidade sistêmica e a saúde como um fenômeno amplo e interdependente, que envolve diferentes sistemas sociais ${ }^{15}$.

Dessa forma, a escolha do pensamento complexo tem o propósito de compreender alguns elementos da multidimensionalidade dos fenômenos que envolvem a utilização dos registros na gerência do cuidado. Com isso, aspirase a um saber não fragmentado, não redutor, reconhecendo que qualquer conhecimento é incompleto, inacabado e requer uma contextualização ${ }^{15}$.

\section{MÉTODO}

Trata-se de um estudo descritivo, com abordagem qualitativa, de caráter compreensivo. Os dados foram coletados na clínica médica de um hospital público de ensino em Minas Gerais. Para a coleta de dados, foram utilizadas: entrevista semiestruturada, observação participante e pesquisa documental, conduzidas pelo pesquisador principal, que estabeleceu uma aproximação prévia com o cenário do estudo. As entrevistas ocorreram entre outubro e novembro de 2017 . Os critérios de inclusão foram: ser enfermeiro do setor citado e atuar na instituição há três meses. Foram excluídos os enfermeiros que estavam de férias, licença médica, licença para estudos na época da coleta de dados e aqueles que, após três tentativas de contato pessoal e/ou telefônico, não se manifestaram para o agendamento da entrevista. Do total de 14 enfermeiros do setor, participaram do estudo 13 enfermeiras.

As entrevistas foram gravadas em meio digital (áudio) e o pesquisador submeteu a transcrição das mesmas à apreciação das participantes a fim de validá-las. Utilizou-se um roteiro sistematizado construído pelos investigadores, contendo as seguintes questões norteadoras: como os enfermeiros compreendem a utilização dos registros de enfermagem para a gerência do cuidado? Quais os tipos de registros utilizados pelos enfermeiros para a gerência do 
cuidado? De que forma os enfermeiros utilizam os registros de enfermagem para a gerência do cuidado? Quais as facilidades e dificuldades para a utilização dos registros de enfermagem na gerência do cuidado?

Concluída a etapa das entrevistas, foi realizada a observação participante, desenvolvida em 21 ocasiões, permeadas por constante interação e diálogo entre o pesquisador e as enfermeiras, totalizando 150 horas. Seguiu-se um roteiro sistematizado em que foram observados: tipo, finalidade dos registros de enfermagem, facilidades e dificuldades da utilização dos registros para a gerência do cuidado em enfermagem. A pesquisa documental ocorreu de forma concomitante à observação e envolveu a análise de prontuários e outros documentos relacionados aos registros de enfermagem, seguindo um roteiro no qual constava: tipo de registro, conteúdo registrado, profissional responsável e finalidade do registro.

As entrevistas foram transcritas em programa Word for Windows e operacionalizadas pelo software Interface de $R$ pourles Analyses Multidimensionnelles de Textes et de Questionnaires (IRaMuTeQ). Do corpus de análise, composto pelas entrevistas, emergiram como produto seis classes a partir da semelhança e dessemelhança dos vocábulos. Com base nas ideias nucleares dessas classes, definiram-se três categorias de análise conforme demonstrado na Figura 1. Os dados obtidos a partir das três técnicas mencionadas organizaram-se separadamente, contudo foram analisados de forma articulada e integrada mediante a análise de conteúdo de Bardin ${ }^{16}$.

\begin{tabular}{|c|c|c|}
\hline Categorias & Classes & Denominação das Classes \\
\hline $\begin{array}{c}\text { Contexto de utilização dos registros de } \\
\text { enfermagem e desenvolvimento da } \\
\text { gerência do cuidado }\end{array}$ & Classe 5 & $\begin{array}{c}\text { Os registros de enfermagem como } \\
\text { ferramenta para a gerência do cuidado }\end{array}$ \\
\cline { 2 - 3 } & Classe 4 & $\begin{array}{c}\text { A inserção dos profissionais de enfermagem } \\
\text { no ambiente da gerência do cuidado }\end{array}$ \\
\hline $\begin{array}{c}\text { Instrumentos adotados na elaboração dos } \\
\text { registros de enfermagem }\end{array}$ & Classe 2 & Os tipos de instrumentos utilizados \\
\cline { 2 - 3 } $\begin{array}{c}\text { Registros de enfermagem como meio de } \\
\text { comunicação }\end{array}$ & Classe 1 & O PE e sua implantação \\
\cline { 2 - 3 } & Classe 3 & $\begin{array}{c}\text { As dificuldades e as facilidades dos registros } \\
\text { de enfermagem para a gerência do cuidado }\end{array}$ \\
\hline
\end{tabular}

FIGURA 1: Categorias emergidas das entrevistas das enfermeiras. Juiz de Fora, MG, Brasil, 2017.

O estudo obedeceu aos preceitos éticos de pesquisas com seres humanos, conforme recomendações da Resolução no 466/2012, do Conselho Nacional de Saúde, tendo sido iniciado após aprovação do Comitê de Ética em Pesquisa e assinatura do Termo de Consentimento Livre e Esclarecido. Para preservar o anonimato das participantes, foi utilizada a codificação ENF seguida de algarismos arábicos conforme a ordem das entrevistas.

\section{RESULTADOS E DISCUSSÃO}

A totalidade dos entrevistados era do sexo feminino. A faixa etária variou de 31 a 40 anos. Quanto ao tempo de formação profissional, oito possuíam de quatro a nove anos e cinco, mais de dez anos. Sete possuíam vínculo institucional de três meses a um ano, demonstrando assim uma equipe de recém-admitidos no hospital. A maioria (dez) possuía pós-graduação lato sensu ou stricto sensu, evidenciando-se o nível de qualificação das profissionais.

O corpus de análise foi constituído por 13 entrevistas, que foram separadas em 742 segmentos de texto (STs), com aproveitamento de 631 STs (85,04\%). Emergiram 25.931 ocorrências (palavras, formas ou vocábulos), sendo 2.667 palavras distintas e 1.289 com uma única ocorrência.

A Figura 2, resultante da análise do IRaMuTeQ, permite visualizar por meio do dendrograma o corpus de análise que se dividiu em seis classes denominadas de acordo com os vocábulos mais frequentes e significativos.

Os STs foram organizados e associados por afinidade, ramificando-se nas seis classes que foram organizadas em três categorias, conforme demonstrado anteriormente na Figura 1.

\section{Categoria 1: Contexto de utilização dos registros de enfermagem e o desenvolvimento da gerência do cuidado}

\section{Classe 5: Os registros de enfermagem como ferramenta para a gerência do cuidado}

Percebeu-se a fragilidade na continuidade do cuidado de enfermagem e falhas nos registros, refletindo em prejuízo na comunicação entre os profissionais de saúde. Além disso, a sobrecarga de atividades resulta em uma gerência do cuidado de enfermagem inadequada e ineficiente:

O registro é muito importante, pois é onde a gente consegue dar uma continuidade no serviço, uma continuidade ao cuidado [...]. Então, hoje os nossos registros de enfermagem às vezes estão um pouco falhos, porque a gente entende também por conta do serviço puxado, tem dias [...] a maior parte dos dias, é puxado[...] (ENF 01). 


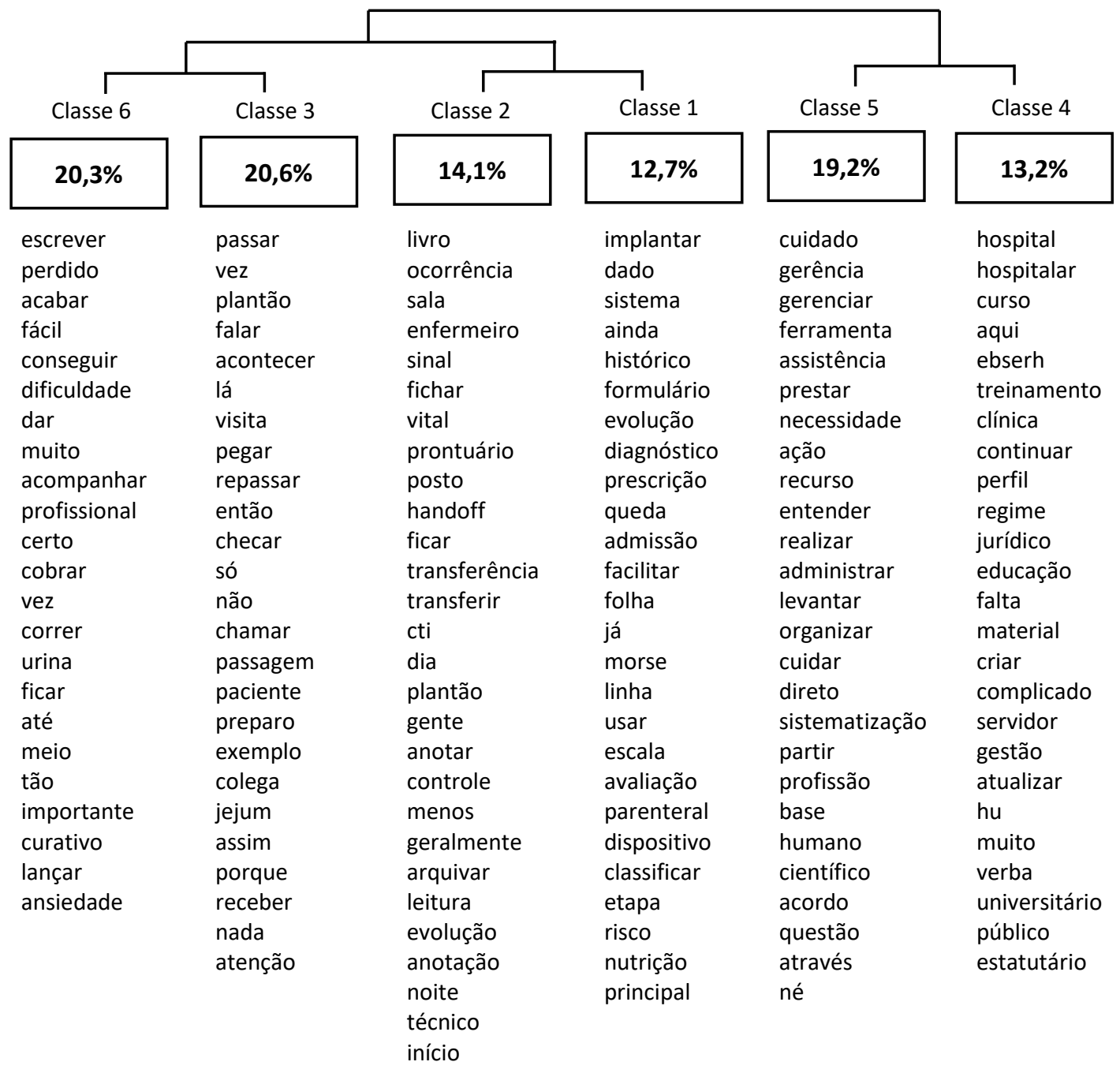

FIGURA 2: Dendograma da Classificação Hierárquica Descendente (CHD) realizado pelo programa IRaMuTeQ. Juiz de Fora, MG, Brasil, 2017.

Conforme o Código de Ética dos Profissionais de Enfermagem, é dever da equipe de enfermagem conceder informações escritas e verbais, completas e verídicas, necessárias à continuidade da assistência e à manutenção da segurança do paciente ${ }^{17}$.

Notou-se uma preocupação das enfermeiras em identificar os problemas apresentados pelo paciente e a importância de registrá-los para uma boa resolubilidade do cuidado e segurança do paciente por meio do registro correto, completo, claro e objetivo:

O registro garante a segurança do paciente, onde tudo deve estar ali registrado corretamente o que foi feito com ele, de forma clara [...]que, às vezes, não adianta escrever, escrever e não ser objetivo, não ser claro (ENF 03).

A baixa qualidade nos registros de enfermagem pode ocasionar problemas graves, que repercutem diretamente na qualidade da assistência e na segurança do paciente, e envolve tanto os profissionais da prática assistencial quanto os gestores, órgãos de classe, pesquisadores e docentes ${ }^{18}$. A adoção de normas e rotinas específicas para as anotações de enfermagem permite padronizar a práticas dos registros, refletindo diretamente na qualidade da assistência, segurança e comunicação ${ }^{19,20}$.

Por meio da pesquisa documental, ratificada nas falas, foi identificado que o prontuário do paciente e os demais documentos, como livros de ocorrência e relatório, são meios que concretizam o processo de cuidado de enfermagem e permitem melhor planejamento da assistência ao paciente: 
A partir dos registros é que vou me organizar, fazer os diagnósticos, planejar esse cuidado. O registro estará ali o tempo inteiro me auxiliando a ter indicadores de assistência para poder fazer um planejamento, seja de um setor, ou um planejamento maior. É ele que vai me dar subsídios para eu fazer uma boa gerência e um bom planejamento desse cuidado (ENF 04).

O enfermeiro recorre aos instrumentos de gestão como ferramentas fundamentais para desenvolver competências além do âmbito clínico. Para isso, ele deve se capacitar teoricamente e aprimorar suas habilidades profissionais, de forma a viabilizar o cuidado e fortalecer os processos relacionais e interativos ${ }^{21}$.

A gerência do cuidado é contextual e relacional, sendo assim complexa; é estabelecida por meio de interações interpessoais produzidas e consumidas no ato de cuidar. Desse modo, requer habilidades sociais dos enfermeiros: comunicação, assertividade e habilidades sociais de trabalho como coordenação e arte de falar em público. Portanto, a prática gerencial envolve aspectos subjetivos além do técnico-científico, pois se trata de um processo social ${ }^{22}$.

O pensamento complexo influencia a gerência do cuidado de enfermagem, pois envolve a constante açãoreflexão-ação, articulação e integração de diversos saberes em busca de um cuidado ampliado, seguro e efetivo ${ }^{14}$. Portanto, deve ser compreendida como um fenômeno complexo marcado por conexões e interconexões humanas com suas singularidades e diversidades biológicas, sociais, culturais e políticas, em constante desorganização e reorganização ${ }^{13,23}$.

\section{Classe 4: A inserção dos profissionais de enfermagem no ambiente da gerência do cuidado}

As participantes citaram alguns temas que necessitavam de aprimoramento para subsidiar a gerência do cuidado de enfermagem, tais como: procedimentos operacionais padrão, gestão hospitalar, liderança, gerenciamento de conflitos e segurança do paciente:

Eu acho que o tema gerenciamento de conflitos é importante, que precisa ser sempre lembrado, atualizado $e$ revisto, porque aqui, tem dia, com plantão com oito profissionais de enfermagem, então cada pessoa pensa de um jeito [...]. Às vezes, nem precisa de um conflito grave, mas, talvez, um conflito por conta de uma escala, folga, como a gente deve gerenciar isso? (ENF 13).

Algumas estratégias e habilidades gerenciais usadas pelos enfermeiros podem auxiliar na resolução dos conflitos: conhecimento técnico-científico, organização, planejamento das ações, trabalho em equipe, flexibilidade, comunicação eficiente, gestão compartilhada, espaços dialógicos que estimulem a integração entre os profissionais e o intercâmbio de saberes ${ }^{24}$. A aproximação entre os membros da equipe e a oportunidade de convívio no trabalho também são fatores importantes para melhora no relacionamento interpessoal ${ }^{25}$. A liderança é uma importante competência para o desenvolvimento da gerência do cuidado, os enfermeiros devem exercer a liderança por meio de exemplo, condução, motivação e estímulo de sua equipe ${ }^{26}$.

Nos momentos de observação participante, constatou-se a existência de capacitações para a equipe de enfermagem, priorizando procedimentos e técnicas, desconsiderando os aspectos que envolvem a articulação entre os instrumentos de gerência e o cuidar. As capacitações podem contribuir para melhorar a qualidade e o entendimento dos registros de enfermagem não apenas como um serviço burocrático, mas como parte essencial do cuidar 8 .19.

A gerência do cuidado de enfermagem envolve múltiplas ferramentas, entre elas os registros, e o pensamento complexo auxilia na compreensão do saber/fazer da enfermagem para transcender o pensamento reducionista, disjuntivo, fragmentador e alcançar um saber/fazer interativo, associativo, unificador ${ }^{27}$.

A necessidade de um curso de aperfeiçoamento sobre a Sistematização da Assistência de Enfermagem (SAE) e o Processo de Enfermagem (PE) foi narrada pelas entrevistadas:

Acho que uma coisa que precisa aprimorar é a parte das prescrições de enfermagem, os planos de cuidados, na verdade, a sistematização da assistência de enfermagem como um todo, sabe, que é uma coisa que a gente aprende na faculdade, mas depois as instituições deixam pra lá (ENF 08).

A implementação do PE requer conhecimento e expertise, para avaliar, diagnosticar, planejar e produzir a assistência de enfermagem voltada às necessidades de saúde dos usuários. Tal conhecimento pode ser apreendido por meio de capacitações permanentes e contribui para a autonomia e visibilidade do enfermeiro ${ }^{28}$.

É inegável que a SAE e o PE são fundamentais para uma assistência qualificada, contudo a concretização dos mesmos ultrapassa questões relacionadas à capacitação profissional. Algumas características institucionais precisam ser analisadas e adequadas para sua implementação, entre elas o dimensionamento de pessoal adequado à quantidade e à complexidade de pacientes, ambiente e recursos materiais favoráveis, valorização profissional, conhecimento e qualidade dos registros ${ }^{29}$. 


\section{Categoria 2: Instrumentos adotados na elaboração dos registros de enfermagem}

\section{Classe 2: Os tipos de instrumentos utilizados}

Por meio da pesquisa documental e entrevistas, foi possível identificar diversificadas modalidades de registros de enfermagem, entre elas: formulário de avaliação do processo de enfermagem, ficha de evolução (follow-up), passante (listagem dos pacientes internados com registro das intercorrências), checklist pré-operatório e livros de ocorrências do setor, exames, registro e controle de passagem de plantão, cultura de vigilância e protocolo.

Os tipos são a evolução de enfermagem, livro de ocorrências, livro de intercorrências do plantão [...]. Além disso, a gente coloca quando o paciente é encaminhado para exame, quando ele volta do exame, quantos pacientes estamos recebendo no início do plantão, quantos a gente está passando (ENF 06).

A criação e a validação de impressos para amparar a sistematização do cuidado de enfermagem são essenciais, pois possibilitam identificar as necessidades humanas e de cuidados; reconhecer problemas; apoiar o processo do raciocínio clínico e detectar indicadores para avaliar as intervenções terapêuticas da equipe de enfermagem ${ }^{30}$. Dessa forma, a utilização dos dados registrados nos instrumentos e a incorporação das informações relevantes para subsidiar a gerência do cuidado sustentam a complexidade desse processo.

\section{Classe 1: O PE e sua implantação}

Algumas enfermeiras relataram os registros de enfermagem provenientes do PE como subsídio para o gerenciamento do cuidado, como ferramenta para o plano de cuidados e avaliação:

O registro é uma ferramenta importante para a sistematização do cuidado, é o conhecimento produzido pela enfermagem, tendo como alicerce a gerência do cuidado, com qualidade (ENF 06).

$O$ registro de enfermagem, como ferramenta proveniente do $\mathrm{PE}$, configura-se como uma tecnologia do cuidado que norteia o raciocínio lógico, qualificando a assistência pela sistematização da avaliação clínica, auxiliando em pesquisa, educação e gerenciamento ${ }^{31}$.

Entretanto, na observação participante, percebeu-se a fragmentação das etapas do $P E$, carência de formulários específicos contemplando todas as suas etapas, o que foi justificado pela atual fase de implantação. Entende-se que esse é um processo complexo visto que o cenário de estudo é um hospital universitário que integra ensino e serviço. A implementação do PE pode facilitar o processo de ensino-aprendizagem com a aplicação da teoria na prática clínica. Sendo assim, o hospital universitário representa um local de modelo para a concretização de aulas práticas da graduação, contribuindo para formação de enfermeiros ${ }^{32}$.

As dificuldades vivenciadas no ensino e na prática da SAE e do PE têm relação com as características operacionais envolvidas na sua realização, o tempo dispensado na elaboração do PE, demandas diversas, fatores organizacionais, instrumentos extensos, além de limitações dos profissionais relacionadas ao conhecimento de semiotécnica/semiologia necessário ao raciocínio clínico e terapêutico ${ }^{33}$.

\section{Categoria 3: Registros de enfermagem como meio de comunicação}

\section{Classe 6: As dificuldades e as facilidades dos registros de enfermagem para a gerência do cuidado}

As dificuldades dos registros de enfermagem para a gerência do cuidado apontadas pelas participantes foram: ausência de evolução de enfermagem, anotações incompletas, falta de clareza nas anotações e tempo dos profissionais, limitação de recursos humanos, número excessivo de pacientes, registros não informatizados e realização do PE de modo incompleto. Estas podem impactar a comunicação da equipe.

Sinto uma dificuldade na falta de um bom registro, que não surja dúvida para toda a equipe de enfermagem. Um bom registro é ele estar claro, bem conciso, bem detalhado, usar palavras e termos que todos conseguem compreender, de preferência utilizar termos técnicos (ENF 12).

Em contrapartida, foram citados aspectos facilitadores da utilização dos registros de enfermagem para a gerência do cuidado, tais como: impressos e formulários de enfermagem, como mecanismos úteis para melhor organização, planejamento e comunicação das atividades diárias da equipe e a continuidade dos registros de enfermagem feitos a cada turno de trabalho.

Um estudo quase-experimental realizado no Rio de Janeiro demonstrou que a implementação de um instrumento formulado a partir de um sistema de linguagem padronizada de diagnósticos, intervenções e resultados de enfermagem direcionado para a área cardiovascular, após a capacitação de enfermeiros, elevou consideravelmente o escore total da qualidade do registro de enfermagem, assim como a avaliação clínica efetuada por enfermeiros na tomada de decisão, no planejamento do cuidado e nos resultados de enfermagem ${ }^{34}$. 
As participantes identificaram que os registros de enfermagem serviram para a otimização do tempo, ordenação dos trabalhos e deram suporte, apoio e orientação à gerência de enfermagem:

Um bom registro ele sempre vai te auxiliar muito no gerenciamento, [...] realizar um bom registro seria uma evolução diária do paciente, completa, inclusive com a sistematização da assistência, todas as intercorrências [...]. Os registros vão auxiliar eu fazer as coisas em um tempo mais certo, reduzir atrasos em alguns procedimentos, ou em alguma outra atividade que eu tenha que realizar, ou um encaminhamento [...] (ENF 04).

Esses dados convergem com os de outro estudo que verificou que o uso de variados registros, como planilhas, sistema de classificação de pacientes, indicadores, entre outros, aliado às competências profissionais como comunicação, organização e planejamento, favorecia as ações da gerência de enfermagem em setores hospitalares $\operatorname{críticos}^{35}$.

\section{Classe 3: A importância dos registros de enfermagem para a passagem de plantão}

Nos discursos das enfermeiras, evidenciou-se que a comunicação na passagem de plantão por meio dos registros de enfermagem, mostrou-se uma importante aliada na condução da gerência do cuidado, serviu para melhorar a integração da equipe multiprofissional, a atualização das informações e o esclarecimento de dúvidas.

Contudo, na observação do cenário de estudo, percebeu-se que a passagem de plantão pelas enfermeiras ocorreu de modo não sistematizado, com relatos de demandas do setor, com pouco ou nenhum registro escrito das intercorrências dos pacientes, apenas relatadas verbalmente. O formulário "passante" serviu como um instrumento acessório que facilitou a condução da passagem de plantão, sem detalhamentos do quadro de saúde do paciente, mas com apontamentos gerais e amplos de seu estado de saúde.

Nesse sentido, um estudo que buscou conhecer a visão dos profissionais de enfermagem sobre a comunicação durante a passagem de plantão e seu impacto na segurança do paciente apontou a necessidade de redução do tempo utilizado, sistematização dos registros para o momento e maior objetividade na transmissão das informações ${ }^{36}$.

\section{Limitações do estudo}

Como limitações do estudo, apontam-se a realização em um único setor hospitalar (clínica médica) e o número restrito de participantes. Logo, os resultados impedem generalizações, fomentando a necessidade de novos estudos em outras áreas hospitalares.

\section{CONCLUSÃO}

Os resultados da pesquisa contribuíram para uma reflexão contemporânea sobre gerência do cuidado de enfermagem na perspectiva do pensamento complexo, para isso se consideraram múltiplas dimensões do uso dos registros. Os registros de enfermagem foram descritos pelas enfermeiras como uma ferramenta para a gerência do cuidado, contudo na prática foi observado que esses registros exerciam o papel protocolar, burocrático associado à gerência de forma desarticulada do cuidado, evidenciando uma relação dicotômica entre gerenciar e cuidar.

Espera-se que este estudo possa contribuir para o serviço e o ambiente acadêmico, estimulando os profissionais a repensarem e refletirem sobre suas práticas, fortalecendo o entendimento dos registros de enfermagem como ferramenta essencial de suporte e avaliação da qualidade da assistência para o enfermeiro realizar a gerência do cuidado no ambiente hospitalar, considerando a SAE e o PE como prioritários nesse processo.

\section{REFERÊNCIAS}

1. Conselho Federal de Enfermagem (COFEN). Guia de recomendações para os registros de enfermagem no prontuário do paciente [Internet]. Brasília (DF): Cofen; 2017. Available from: http://biblioteca.cofen.gov.br/guia-de-recomendacoes-pararegistro-de-enfermagem-no-prontuario-do-paciente-e-outros-documentos-de-enfermagem/.

2. Figueiredo T, Silva PLN, Guimarães LF, Guimarães CF, Oliveira MKS, Alves ECS. Assessment of Nursing Records of Patients Admitted to the Medical Clinic of a University Hospital from the Northern Region of Minas Gerais State. J. res.: fundam. care. online [Internet] 2019 [cited 2020 June 06]; 11(2):390-6. DOI: https://doi.org/10.9789/2175-5361.2019.v11i2.390-396.

3. Carneiro SM, Dutra HS, Costa FM, Mendes SE, Arreguy-Sena C. Use of abbreviations in the nursing records of a teaching hospital. Rev. Rene (Online). 2016 [cited 2020 May 21]; 17(2):208-16. DOI: https://doi.org/10.15253/21756783.2016000200008.

4. Gomes DC, Cubas MR, Pleis LE, Shmeil MAH, Peluci APVD. Terms used by nurses in the documentation of patient progress. Rev. Gaúch. Enferm. [Internet]. 2016 [cited 2020 June 06]; 37(1): e53927. DOI: http://dx.doi.org/10.1590/19831447.2016.01.53927.

5. Silva TGD, Santos RM, Crispim LMC, Almeida, LMWS. The content of nursing records in hospitals: contributions to the development of the nursing process. Enferm. Foco. 2016 [cited 2020 May 21]; 7(1):24-27. DOI: https://doi.org/10.21675/2357707X.2016.v7.n1. 
6. Krauzer IM, Karal A, Bordignon M, Trindade LL. Records of nursing from the perspective of an integrative review. J. nurs. health. (Online). 2015 [cited 2020 May 21]; 5(1):68-79. Available from: https://periodicos.ufpel.edu.br/ojs2/index.php/enfermagem/article/view/3866.

7. Dutra HS, Mendes SE, Carneiro SM, Costa FM, Barboza RCP, Ribeiro LC. Nursing records at a teaching hospital: a quasiexperimental study. Online braz. j. nurs. (Online). 2016 [cited 2020 May 21]; 15(3): 351-360. DOI: https://doi.org/10.17665/1676-4285.20165470.

8. Barreto JA, Lima GGD, Xavier CF. The inconsistency of nursing records in audit process la incompatibilidad de los registros de enfermería em processo de auditoría. Rev. enferm. Cent.-Oeste Min. 2016 [cited 2020 May 21]; 1(6): 2081-2093. DOI: https://doi.org/10.19175/recom.v0i0.917.

9. Mutshatshi TE, Mothiba TM, Mamogobo PM, Mbombi MO. Record-keeping: Challenges experienced by nurses in selected public hospitals. Curationis. 2018 [cited 2020 June 06]; 41(1): 1931. DOI: https://doi.org/10.4102/curationis.v41i1.1931.

10. Lanzoni GMM, Magalhães ALP, Costa VT, Erdmann AL, Andrade SR, Meirelles BHS. Becoming nursing manager in the nested and complex border of caring and management dimensions. Rev. eletrônica enferm. 2015 [cited 2020 May 21]; 17(2): 322-32. DOI: http://dx.doi.org/10.5216/ree.v17i2.29570.

11. Siewert JS, Rodrigues DB, Malfussi LBH, Andrade SR, Erdmann AL. Management of Integral Care in Nursing: reflections under the perspective of complex thinking. Reme, rev. min. enferm. 2017 [cited 2020 May 21]; 21: e-1047. DOI: https://doi.org/10.5935/1415-2762.20170057.

12. Cassiani SHDB, Bassalobre-Garcia A, Reveiz L. Universal Access to Health and Universal Health Coverage: identification of nursing research priorities in Latin America. Rev. Latino-Am. Enfermagem. 2015 [cited 2020 May 21]; 23(6):1195-1208. DOI: http://dx.doi.org/10.1590/0104-1169.1075.2667.

13. Morin E. Introdução ao pensamento complexo. 5a ed. Porto Alegre (RS): Sulina; 2015.

14. Cruz RAO, Araujo ELM, Nascimento NM, Lima RJ, França JRFS, Oliveira J. Reflections in the light of the complexity theory and nursing education. Rev. Bras. Enferm. [Internet]. 2017 Feb. [cited 2020 June 06]; 70(1): 236-239. DOI: https://doi.org/10.1590/0034-7167-2016-0239.

15. Backes DS, Zamberlan C, Colomé J, Souza MT, Marchiori MT, Erdmann AL, Maya AMS. Systemic Interactivity between Interdependent Concepts of Nursing Care. Aquichan [online]. 2016 [cited 2020 June 06]; 16(1): 24-31. DOI: http://dx.doi.org/10.5294/aqui.2016.16.1.4.

16. Bardin, L. Análise de Conteúdo. Tradução de Luís Antero Reto e Augusto Pinheiro. São Paulo: Edições 70; 2016.

17. Conselho Federal de Enfermagem (COFEN). Resolução COFEN no 564, de 6 de novembro de 2017. Aprova o novo Código de Ética dos Profissionais de Enfermagem. Brasília (DF): Conselho Federal de Enfermagem; 2017. Available from: http://www.cofen.gov.br/resolucao-cofen-no-5642017_59145.html.

18. Melo LS, Figueiredo LS, Pereira JM, Flores PV, Cavalcanti AC. Effect of an educational program on the quality of Nursing Process recording. Acta Paul. Enferm. 2019 [cited 2020 June 06]; 32(3):246-53. DOI: http://dx.doi.org/10.1590/1982-0194201900034.

19. Borges FFD, Azevedo CT, Amorim TV, Figueiredo MAG, Ribeiro, RGM. Importance of nursing records according to nursing team: professionals and institutional implications. Rev. enferm. Cent.-Oeste Min. 2017 [cited 2020 June 06], 7(1): 1-8. DOI: https://doi.org/10.19175/recom.v7i0.1147.

20. Hardiker NR, Dowding D, Dykes PC, Sermeus W. Reinterpreting the nursing record for an electronic context. Int. J. Med. Inform. 2019 [cited 2020 June 06]; 127: 120-126. DOI: https://doi.org/10.1016/j.ijmedinf.2019.04.021.

21. Martins PF, Perroca MG. Care necessities: the view of the patient and nursing team. Rev. Bras. Enferm. [Internet]. 2017 [cited 2020 May 21]; 70(5): 1080-6. DOI: http://dx.doi.org/10.1590/0034-7167-2016-0197.

22. Montezeli JH, Haddad MCFL, Garanhani ML, Peres AM. Improving social skills in care management provided by nurses: intervention research. Rev. Bras. Enferm. [Internet]. 2019 [cited 2020 Sep 17]; 72(Suppl 1):49-57. DOI: http://dx.doi.org/10.1590/0034-7167-2017-0918.

23. Morin E. O método 2: a vida da vida. Porto Alegre (RS): Sulina; 2001.

24. Martins FZ, Dall'Agnol CM. Surgical center: challenges and strategies for nurses in managerial activities. Rev. Gaúch. Enferm. [Internet]. 2016 [citado 2020 June 06]; 37(4): e56945. DOI: http://dx.doi.org/10.1590/1983-1447.2016.04.56945.

25. Pinhatti EDG, Vannuchi MTO, Sardinha DSS, Haddad MCL. Job rotation of nursing professionals among the sectors of a hospital: a management tool in conflict resolution. Texto Contexto Enferm. [Internet]. 2017 [citado 2020 June 06]; 26(2): e1180015. DOI: http://dx.doi.org/10.1590/0104-07072017001180015.

26. Farah BF, Dutra HS, Sanhudo NF, Costa LM. Perception of nurse supervisors on leadership in primary care. Rev. cuid. (Online) 2017 [cited 2020 May 21]; 8(2): 1638-55. DOI: http://dx.doi.org/10.15649/cuidarte.v8i2.398.

27. Backes MTS, Carvalho KM, Santos EKA, Backes DS. New coronavirus: what does nursing have to learn and teach in times of a pandemic? Rev. Bras. Enferm. [Internet]. 2020 [cited 2021 May 17]; 73(Suppl 2): e20200259. DOI: https://doi.org/10.1590/0034-7167-2020-0259.

28. Benedet SA, Padilha MI, Peres MAA, Bellaguarda MLR. Essential characteristics of a profession: A historical analysis focusing on the nursing process. Rev. Esc. Enferm. USP. [Internet]. 2020 [citado 2020 Sept. 06]; 54:e03561. DOI: https://doi.org/10.1590/S1980-220X2018047303561.

29. Soares MI, Resck ZMR, Terra F de S, Camelo SHH. Systematization of nursing care: challenges and features to nurses in the care management. Esc. Anna Nery [Internet]. 2015 Mar. [cited 2020 June 27]; 19(1): 47-53. DOI: https://doi.org/10.5935/14148145.20150007. 
30. Arreguy-Sena C, Marques TO, Souza LC, Martins NA, Krempser P, Braga LM, Parreira PMSD. Construction and validation of forms: systematization of the care of people under hemodialysis. Rev. Bras. Enferm. [Internet]. 2018 [cited 2020 May 21]; 71(2): 379-9. DOI: https://doi.org/10.1590/0034-7167-2015-0130.

31. Silveira DM, Nicola GDO, Chagas MC, Pereira FW, Ilha S, Magalhães M. Public university students' ease and/or difficulties in preparing nursing progress notes. Rev. Enferm. UERJ [Internet]. 2015 [cited 2020 May 21]; 23(6): 832-7. DOI: https://doi.org/10.12957/reuerj.2015.7267.

32. Benedet SA, Padilha MI, Gelbke FL, Bellaguarda MLR. The model professionalism in the implementation of the Nursing Process (1979-2004). Rev. Bras. Enferm. [Internet]. 2018 [citado 2020 Sept. 06];71(4):1907-14. DOI: http://dx.doi.org/10.1590/00347167-2017-0226.

33. Gutiérrez MGR, Morais SCRV. Systematization of nursing care and the formation of professional identity. Rev. Bras. Enferm. [Internet]. 2017 [cited 2020 May 21]; 70(2): 455-60. DOI: http://dx.doi.org/10.1590/0034-7167-2016-0515.

34. Belém ARSC, Figueiredo LS, Pereira JMV, Flores PVP, Cavalcanti ACD. Effect of a standardized instrument on the quality of nurses' records: a quasi-experimental study. Reme, rev. min. enferm. 2019 [cited 2020 June 06]; 23:e-1252. DOI: http://dx.doi.org/10.5935/1415-2762.20190100.

35. Vasconcelos RO, Bohrer CD, Rigo DFH, Marques LGS, Oliveira JLC, Tonini NS, Nicola AL. Means for nursing management used in critical hospital units. Enferm. Foco. 2016 [cited 2020 June 06]; 7 (3/4): 56-60. Available from: http://revista.cofen.gov.br/index.php/enfermagem/article/view/944.

36. Silva MF, Anders JC, Rocha PK, Souza AIJ, Burciaga VB. Communication in nursing shit handover: pediatric patient safety. Texto Contexto Enferm. 2016 [cited 2020 May 21]; 25(3): e3600015. DOI: http://dx.doi.org/10.1590/0104-07072016003600015. 